\title{
DÜBLIN
}

Technological University Dublin

ARROW@TU Dublin

\section{Ray-trace Modelling of Reflectors for Quantum Dot Solar Concentrators}

\author{
Manus Kennedy \\ Technological University Dublin, manus.kennedy@tudublin.ie \\ Sarah McCormack \\ Technological University Dublin \\ John Doran \\ Technological University Dublin, john.doran@tudublin.ie
}

See next page for additional authors

Follow this and additional works at: https://arrow.tudublin.ie/dubencon2

Part of the Engineering Commons

\section{Recommended Citation}

Kennedy, M., McCormack, S. \& Doran, J. (2007). Ray-trace modelling of reflectors for quantum dot solar concentrators. Proceedings of SPIE Optics and Photonics and Solar Energy Conference, San Diego, California, USA, 26-30, August. doi:10.1117/12.733786

This Conference Paper is brought to you for free and open access by the Dublin Energy Lab at ARROW@TU Dublin. It has been accepted for inclusion in Conference Papers by an authorized administrator of ARROW@TU Dublin. For more information, please contact arrow.admin@tudublin.ie, aisling.coyne@tudublin.ie, gerard.connolly@tudublin.ie.

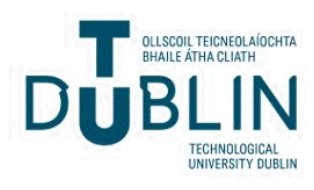


Authors

Manus Kennedy, Sarah McCormack, John Doran, and Brian Norton

This conference paper is available at ARROW@TU Dublin: https://arrow.tudublin.ie/dubencon2/18 


\title{
Ray-trace modelling of reflectors for quantum dot solar concentrators
}

\author{
M. Kennedy ${ }^{*}$, S. J. McCormack, J. Doran, B. Norton \\ Dublin Energy Lab., Focas Institute, School of Physics, Dublin Institute of Technology, Kevin St, \\ Dublin, Ireland.
}

\begin{abstract}
Quantum Dot Solar Concentrators (QDSCs) are static, non-imaging concentrators which do not require expensive solar tracking and concentrate both direct and diffuse light. Optical efficiencies $\left(\eta_{\mathrm{opt}}\right)$ and concentration ratios (C) of a single plate QDSC were calculated by Monte-Carlo ray-trace modelling. Consideration of reflection, refraction, quantum dot (QD) photon emission and absorption and light attenuation in the device matrix were included in the analysis. In this paper, the effect of placing plane and diffuse reflectors at the rear surface was analyzed. Mirrors with a structured surface (saw-tooth shaped) were also modelled and the effect of each reflector type on $\mathrm{C}$ was determined, for direct and diffuse incident light. The diffuse and structured reflectors perform better than the plane reflector under direct light, but there is no significant difference under diffuse light. A spectrally selective reflector, placed at the top surface, reflects light emitted by QDs inside the escape cone back into the concentrator. For a particular set of model parameters, the model results show an increase in $\mathrm{C}$ of $13 \%$ due to the inclusion of a spectrally selective reflector.
\end{abstract}

Keywords: Quantum Dot, Luminescent Solar Concentrator, Static Non-imaging Diffuse Concentrator, Ray-trace Modelling,

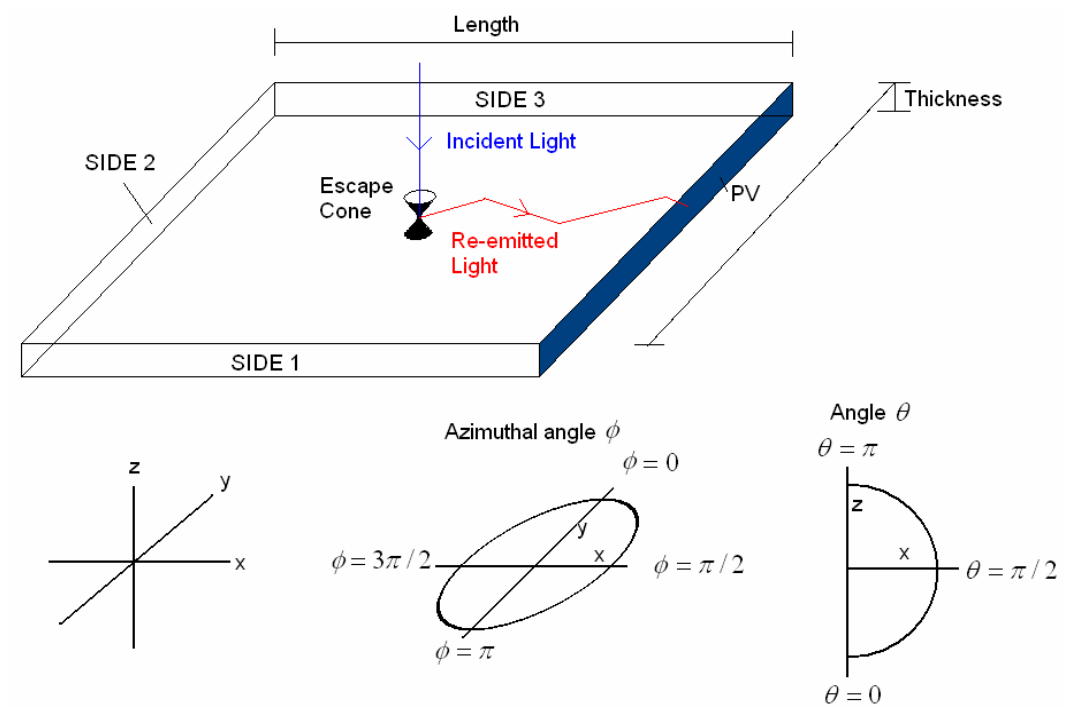

Figure 1. Luminescent solar concentrator (LSC) consisting of a transparent polymer plate doped with luminescent dye, with a PV cell attached to one side. A Quantum Dot Solar Concentrator is an LSC that employs quantum dots rather than a luminescent dye. Also shown are the definitions of angle and azimuth angle used in the model.

\footnotetext{
* Corresponding author Manus Kennedy, Dublin Energy Lab., Focas Institute, DIT - Kevin Street, Tel: +353 1 4027967, Email: manus.kennedy@dit.ie
} 


\section{INTRODUCTION}

\subsection{Luminescent Solar Concentrators}

One approach to increasing the economic viability of photovoltaic (PV) cells for electricity generation is the concentration of incident solar energy. Luminescent solar concentrators (LSCs) ${ }^{1}{ }^{2}$ are static, non-imaging concentrators which do not require expensive solar tracking and can concentrate both direct and diffuse light, a significant advantage in Northern European climates where $>50 \%$ of total annual solar irradiation is diffuse. As incident insolation passes through an LSC device matrix, consisting of a flat polymer plate doped with a luminescent dye as shown in Figure 1, it is absorbed by the dye. Longer wavelength light is subsequently emitted isotropically by the luminescent dye. The refractive index of the LSC is larger than that of the surrounding air, resulting in much of the emitted light being trapped by total internal reflection (TIR). Mirrors are placed adjacent and parallel to the rear surface and sides 1, 2 and 3 (side numbers denoted in Figure 1). These mirrors reflect light that may be incident on these surfaces outside the angular range for TIR. There is a small air-gap between these mirrors and the device matrix. Light emitted in the device matrix is transmitted to one edge, where a PV cell is attached. A Quantum Dot Solar Concentrator ${ }^{3}$ (QDSC) is an LSC that employs QDs rather than a luminescent dye.

\subsection{Rear Reflectors}

The use of a rear reflector allows incident light a double pass through the device and, therefore, a higher probability that an incident photon will be absorbed. Plane, diffuse and structured rear reflectors have been modelled and concentration ratios were calculated for each reflector type under direct and diffuse incident light. Figure 2 shows each reflector type under direct incident light. There are two advantages in including a diffuse rear reflector instead of a plane reflector, as illustrated in Figure 2. (1) Direct light is reflected at an angle thereby increasing the pathlength on the second pass up through the device, allowing more light to be absorbed in the plate. (2) A small fraction of incident light, which enters the device close to the PV cell, will be reflected directly onto the cell by the diffuse reflector but not by the plane reflector. A structured rear reflector consisting of convex curved surfaces was proposed by Reisfeld et al. ${ }^{4}$. A simplified 'saw-tooth' structured reflector, as shown in Figure 2, is considered here. Such a reflector would also result in advantages 1 and 2 outlined above.

Using direct incidence, significant increases have been measured in the power output of LSCs and QDSCs with a diffuse rear reflector compared to the same device with a plane reflector ${ }^{567}$. DeCardona et al. ${ }^{7}$ measured no increase with the diffuse reflector under cloudy diffuse conditions. In this paper, the ray-trace model is used to determine if diffuse or structured rear reflectors perform better than a plane rear reflector under direct and diffuse incident light.

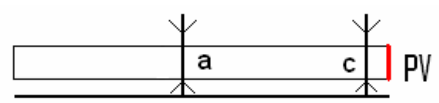

Plane reflector
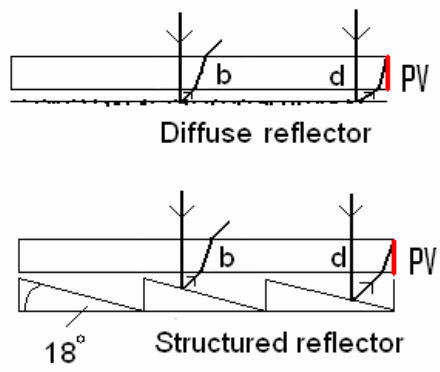

Figure 2. For direct incidence, there is an increased average pathlength using a diffuse or structured reflector (b) compared to a plane reflector (a). The increased pathlength results in more incident light being absorbed for a given QD concentration. Also, for direct incidence, there is a chance that light incident close to the PV cell will be reflected directly onto the PV cell using a diffuse or structured reflector (d), but not using a plane reflector (c). 


\subsection{Spectrally Selective Reflectors}

For a QDSC described by the parameters in section 2, escape cone losses account for 45\% of photons emitted by QDs. Spectrally selective reflecting top layers 89101112 can reduce the escape-cone losses in the device by reflecting light in the QD emission range, while allowing (almost all) light in the QD absorption range to enter the device. Section 3 gives predicted C values for a QDSC with and without a spectrally selective reflector (SSR) with near ideal properties.

\section{RAY TRACE MODEL AND INPUT PARAMETERS}

Monte-Carlo ray-trace modelling is used to determine the optical efficiency $\left(\eta_{\text {opt }}\right)$ of an LSC device ${ }^{513} 141516 . \eta_{\text {opt }}$ is defined as the fraction of photons transmitted to the PV cell, compared to those incident on the concentrator top surface;

$\eta_{\mathrm{opt}}=\frac{\text { total photons transmitted to PV }}{\text { total photons incident on top surface }}$

The concentration ratio $(\mathrm{C})$ is given by;

$\mathrm{C}=\eta_{\text {opt }} \mathrm{G}_{\text {geom }}$

The Geometric gain, $\mathrm{G}_{\text {geom }}$, is defined as the concentrator plate top surface area, $\mathrm{A}_{\text {conc }}$, divided by the total PV area, $\mathrm{A}_{\mathrm{pv}}$;

$\mathrm{G}_{\text {geom }}=\mathrm{A}_{\text {conc }} / \mathrm{A}_{\mathrm{pv}}$

To model a QDSC, a photon is traced through the QDSC until it is lost from the system or transmitted to the PV. The loss mechanisms included are escape-cone losses, matrix attenuation losses, quantum dot (QD) quantum yield (QY) losses, side mirror reflection losses, and initial top surface reflection losses. A wavelength independent matrix attenuation coefficient of $0.02 \mathrm{~cm}^{-1}$, and a refractive index of 1.5 were assumed. The QD QY, defined as the ratio of photons emitted by a QD relative to the number of photons absorbed, was variable. The QDSC dimensions were $11 \times 11 \times 0.3 \mathrm{~cm}$. The wavelength independent reflection coefficients of the plane, diffuse and structured reflecting surfaces were assumed to be 0.94. A diffuse incident light angular distribution was approximated by selecting, at random, incident angles and azimuth angles (defined in Figure 1) from $0 \rightarrow \pi / 2$ and from $0 \rightarrow 2 \pi$, respectively.

\section{RESULTS}

\subsection{Rear Reflectors}

Using QD absorption spectrum 2 and the QD emission spectrum, shown in Figure 3, C was calculated for direct incident light using the plane, diffuse and structured rear reflectors. The AM 1.5 solar spectrum (from $400 \mathrm{~nm}$ to $950 \mathrm{~nm}$ ) was used as the incident light spectrum. For direct incidence, there is an increase in $\eta_{\text {opt }}$ (and hence in C) when using the diffuse and structured reflectors compared to the plane reflector as the initial pathlength of incident light is longer and therefore more incident light is absorbed. Also, a small fraction of incident light, which enters the device close to the PV cell, will be reflected directly onto the cell by the diffuse and structured reflectors but not by a plane reflector, as illustrated in Figure 2. Figure 4 shows predicted $\mathrm{C}$ for each type of reflector for a range of QD QYs, with the diffuse and structured reflectors attaining higher $\mathrm{C}$ than the plane reflector. For devices with low QD QY there is a large relative increases in $\mathrm{C}$ when using the diffuse and structured reflectors compared to the plane reflector. For example, with a $\mathrm{QD}$ QY of $10 \%, C$ obtained using the diffuse reflector $(C=0.38)$ is double that attained with the plane reflector $(C=0.19)$. At the high QD QYs required for a useful QDSC, the addition of a diffuse/structured rear reflector results in a much smaller relative increase in $\mathrm{C}$.

With diffuse incident light there is no significant difference in using the different types of rear reflectors, for any QD QY, as shown in Figure 5. More than 50\% of total annual solar irradiation in the Northern European climate is diffuse. 
On a clear day, light is only incident directly (i.e. perpendicular to the top surface) for a short part of the day. Therefore, of the two angular distributions of incident light considered (i.e. direct and diffuse), the predicted results for diffuse incidence are more relevant. With predicted $\mathrm{C}$ being so similar for each reflector type (in the case of diffuse incidence) the actual reflection coefficient of the reflector used, along with the cost, are more important considerations than the type of reflector used.

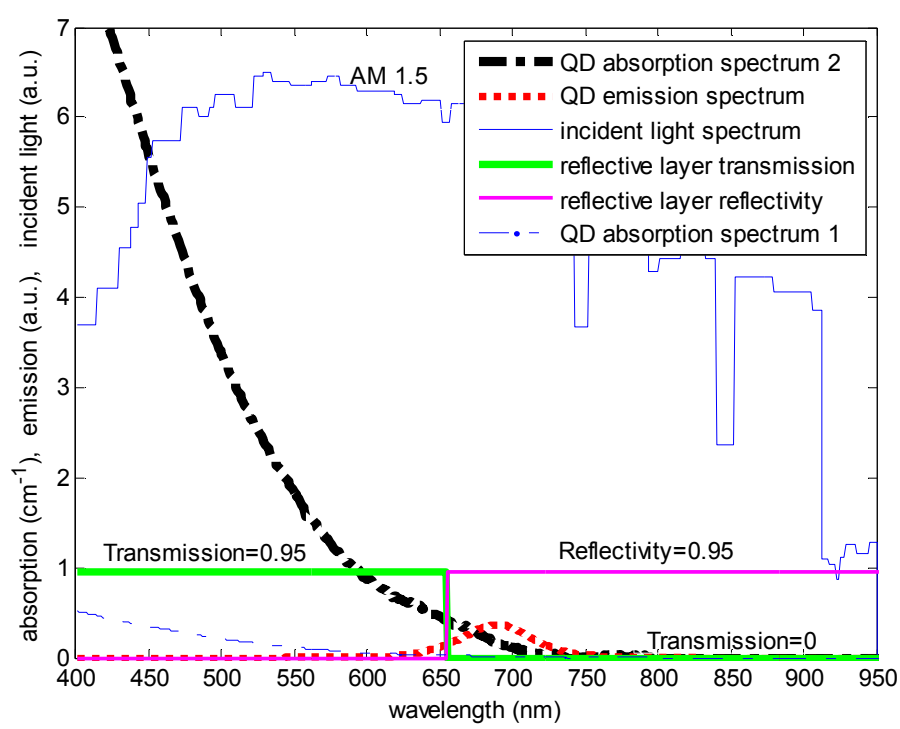

Figure 3. QD Absorption spectrum 1 and the emission spectrum are measured spectra of CdSe QDs (with CdS/CdZnS/ZnS coating, fabricated at Utrecht University). Absorption spectrum 2, corresponding to a higher QD concentration, is extrapolated from QD absorption spectrum 1. Also shown are the AM 1.5 solar spectrum $(400-950 \mathrm{~nm})$ and the transmission and reflectivity of a spectrally selective reflector with near ideal properties.

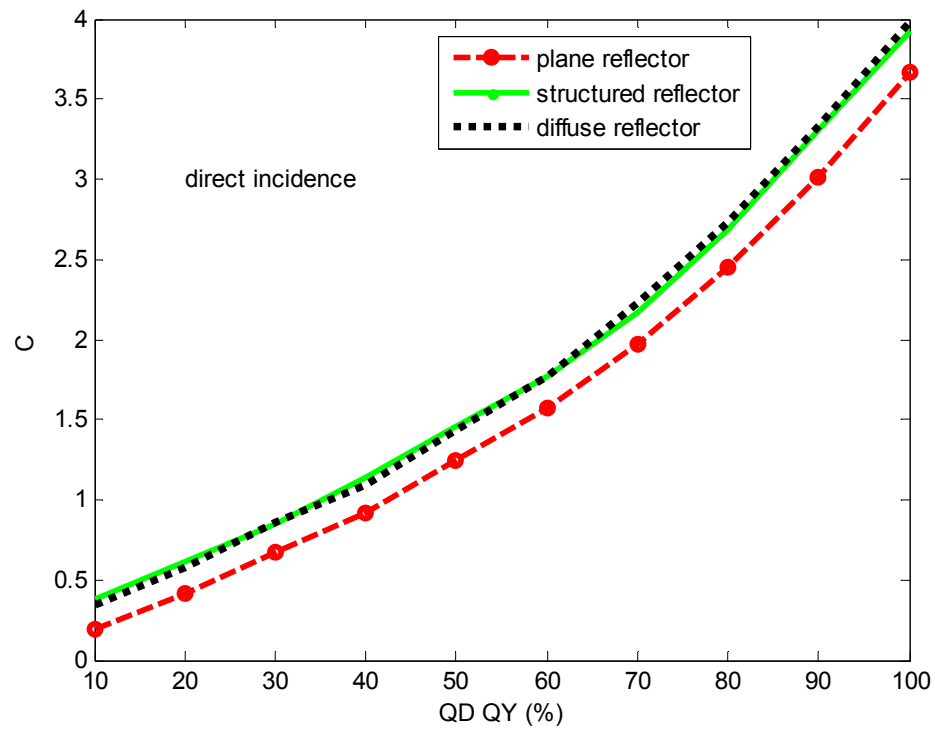

Figure 4. Variation of C with QD QY, for direct incident light. Higher C are achieved using the diffuse and structured reflectors, compared to the plane reflector. 


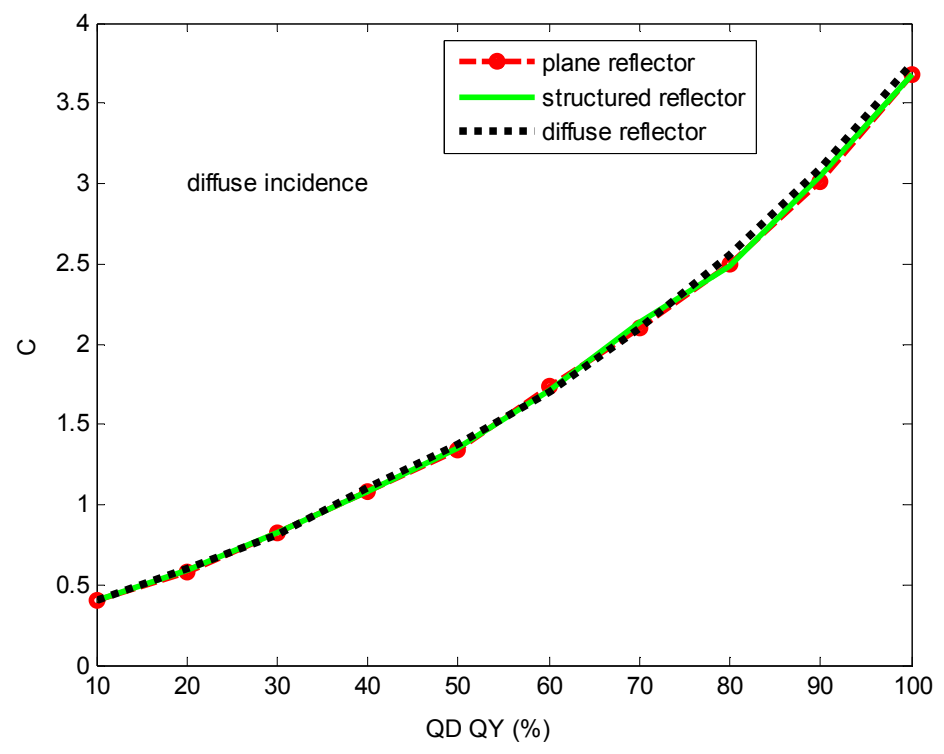

Figure 5 Variation of $\mathrm{C}$ with QD QY, for diffuse incident light. There is no significant difference in predicted C values, using the diffuse or structured reflector, compared to the plane reflector.

\subsection{Spectrally Selective Reflecting Top Layer}

Figure 6 shows the fraction of rays reaching the PV (optical efficiency, $\eta_{\text {opt }}$ ) and the loss mechanisms in the device for diffuse incident light. It was assumed that the QD QY was 100\%. 14\% of total incident light was reflected from the top surface. Due to the limited absorption range of the QDs (up to $\sim 690 \mathrm{~nm}$ ), 46\% of total incident light entered the device but was not absorbed, leaving only $40 \%(100 \%-46 \%-14 \%)$ absorbed in the device. Escape cone losses account for, at least, $18 \%$ of total incident light or $45 \%(18 \% / 40 \%)$ of light emitted by QDs. In order to reduce the escape cone losses, a spectrally selective reflector (SSR) may be placed at the top surface. The SSR is assumed to have a transmission of $95 \%$ at wavelengths shorter than the cut-off wavelength. It is assumed to have a reflectivity of $95 \%$ and $0 \%$ at wavelengths longer and shorter than the cut-off wavelength, respectively, as shown in Figure 3. The reflectivity and transmission coefficients are assumed to be independent of the angle of incidence.

Figure 7 shows the fraction of rays reaching the PV and the device loss mechanisms assuming a QD QY of $100 \%$ and the SSR placed at the top surface, with a small air gap between the SSR and the device matrix. The SSR transmission loss and initial reflection loss from the matrix top surface accounted for 54\% of total incident light. Even with the SSR, escape cone losses still account for $6 \%$ of total incident light (18\% of light emitted by QDs). Light emitted outside the angular range for TIR is reflected back by the SSR at a small angle. Therefore, the pathlength to the PV is likely to be long and there will be multiple reflections at the top and rear reflectors before reaching the PV. The multiple reflections required to reach the PV explains the escape cone losses of $6 \%$ and why the rear reflector reflection losses are higher when the SSR is added. The matrix attenuation losses are also higher due to the long pathlength to the PV of photons reflected by the SSR. Even though the reflectivity of the SSR is high (0.95) and an ideal cut-off between transmission and reflection was assumed, the relative increase in the fraction of rays reaching the PV, compared to the case without the SSR, is just $13 \%$. Figure 8 shows the predicted C values for varying QD QY. With QD QY $<70 \%$, the SSR results in a decrease in $\eta_{\mathrm{opt}}$ and hence in $\mathrm{C}$. While further modelling (and experimental) results are required to quantify the effectiveness of a spectrally selective reflecting top layer, it is clear from the model results to date that very high SSR reflection coefficients will be required for such a layer to be an advantage. 


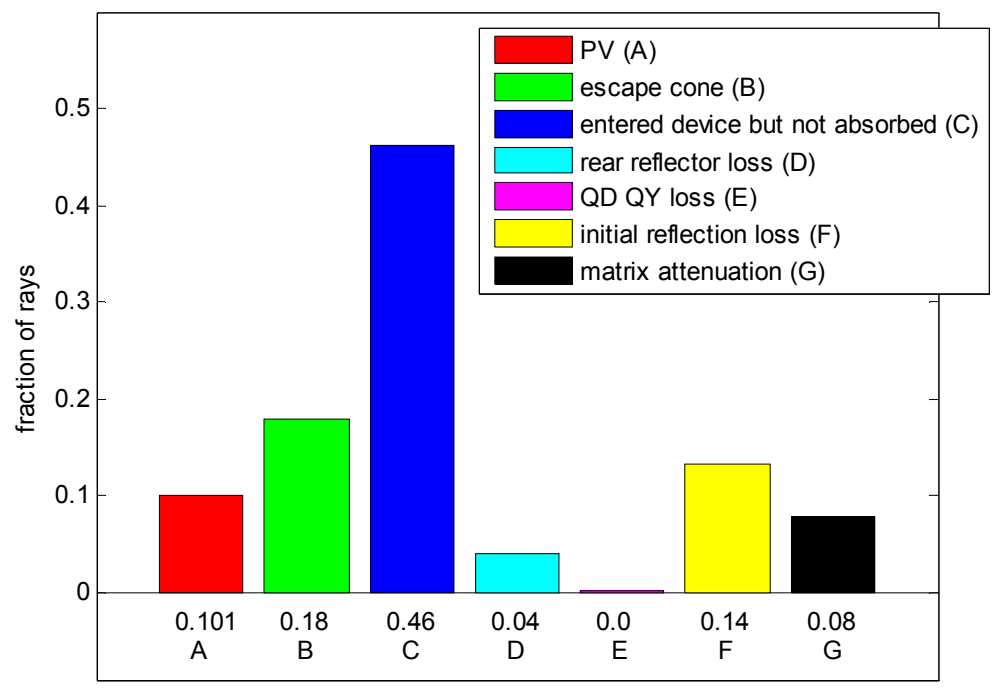

Figure 6. Fraction of incident rays reaching PV (optical efficiency) and loss mechanisms for a device without the spectrally selective reflector. QD QY $=100 \%$. The high fraction not absorbed results from the limited absorption range of the QDs. $18 \%$ of total incident light (45\% of light emitted by QDs) is lost through the escape cone at the top surface.

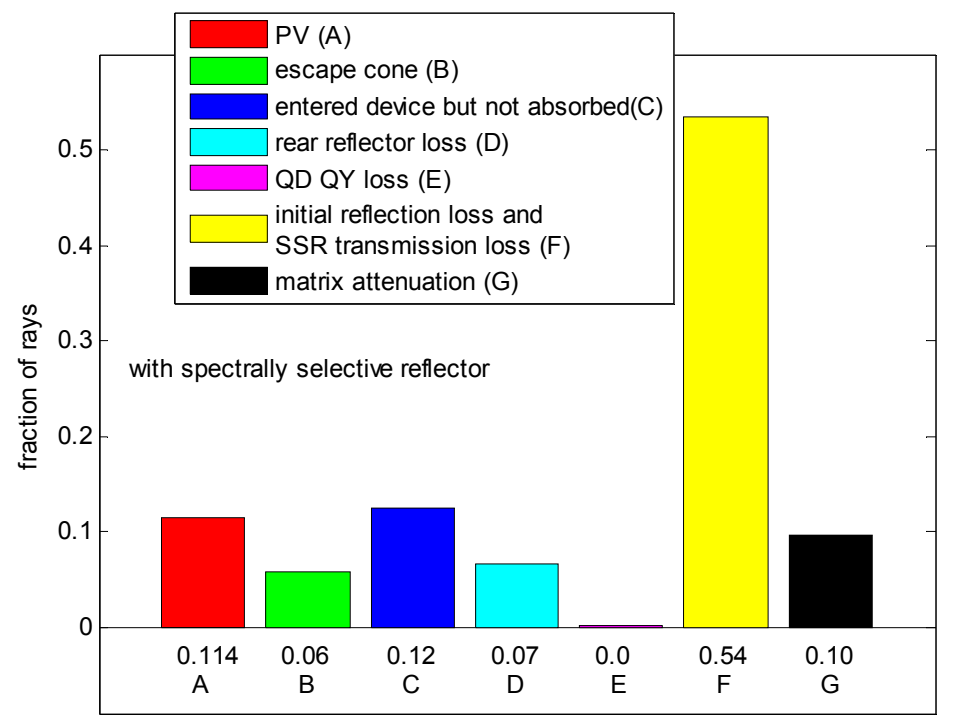

Figure 7 Fraction of incident rays reaching PV (optical efficiency) and loss mechanisms for a device with the spectrally selective reflector (SSR). QD QY $=100 \%$. The SSR reflects light at wavelengths longer than the SSR cut-off wavelength (which includes some incident light inside the QD absorption range). Matrix attenuation and rear reflector losses are higher compared to Figure 6. Escape cone losses are lower, however, resulting in an increased optical efficiency, compared to Figure 6. 


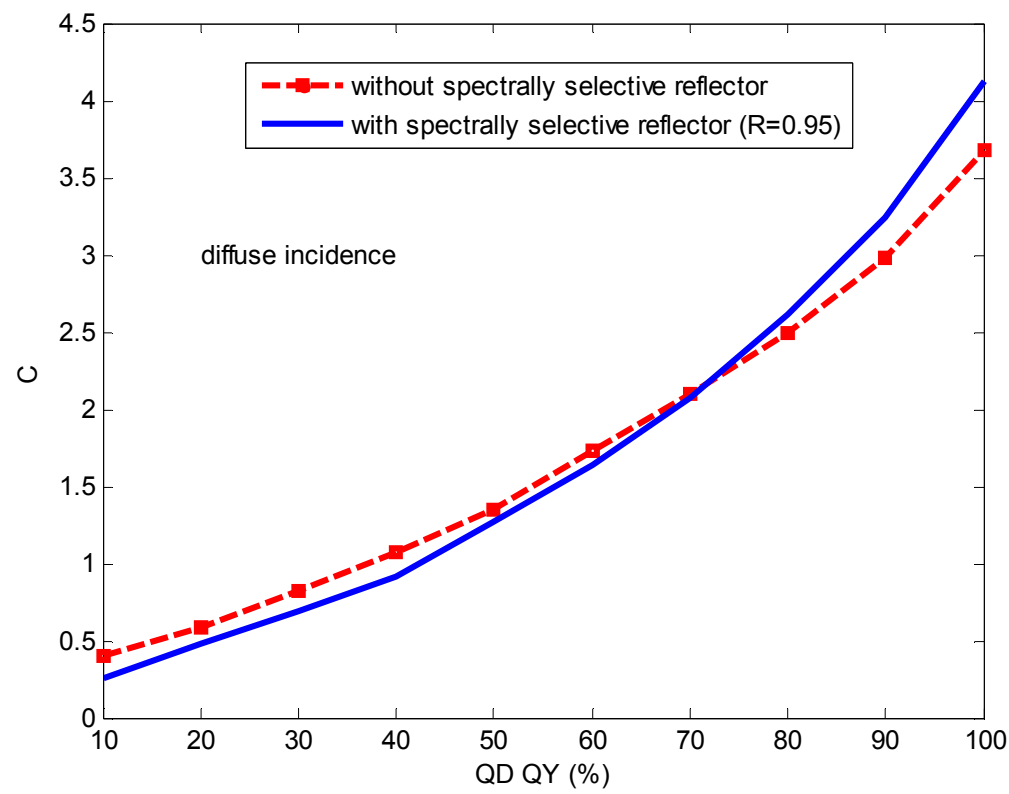

Figure 8. Variation of C with QD QY. At high QD QY (>70\%), the spectrally selective reflector (SSR) results in an increase in optical efficiency and C. At low QD QY, however, the SSR actually results in a decrease in C.

\section{CONCLUSION}

Using Monte-Carlo ray-trace modelling, optical efficiencies and concentration ratios $(\mathrm{C})$ have been predicted for an $11 \times 11 \times 0.3 \mathrm{~cm}$ QDSC incorporating plane, diffuse and structured external rear reflectors. Reflection coefficients of 0.94 were assumed for the surfaces of each reflector type. The diffuse and structured reflectors obtained the highest $\mathrm{C}$ under direct light. For diffuse incidence, there was no significant difference in predicted $\mathrm{C}$ using the three types of rear reflector, indicating that the actual reflection coefficient of the reflector used, along with the cost, are more important considerations than the type of reflector used.

In the device modelled, escape cone losses accounted for $45 \%$ of all photons emitted by QDs. A near ideal spectrally selective reflector (SSR) has been modelled, with a reflection coefficient of 0.95 . The SSR reduces the escape-cone losses in the device by reflecting light in the QD emission range, while allowing (almost all) light in the QD absorption range to enter the device. The SSR was assumed to have an ideal cut-off between reflection and transmission and to have reflection and transmission properties independent of the angle of incidence. Escape cone losses remain, even with the SSR, due to the imperfect SSR reflectivity (0.95). Predicted C are $13 \%$ higher (for QD QY=100\%) with the SSR than without.

\section{ACKNOWLEDGEMENTS}

The authors would like to acknowledge the collaboration of the EU-funded FULLSPECTRUM group, especially R. Koole of Utrecht University for supplying QD absorption and emission spectra. The Focas Institute is funded by the Irish Higher Education Authority with assistance from the European Regional Development Fund. 


\section{REFERENCES}

1. W. H. Weber, J. Lambe, "Luminescent greenhouse collector for solar radiation,” Applied Optics, 15, 2299-2300, 1976.

2 A. Goetzberger, W. Greubel, "Solar Energy Conversion with Fluorescent Collectors," Applied Physics, 14, 123-139, 1977.

3 K.W.J. Barnham, J.L. Marques, J. Hassard, P. O'Brien, "Quantum dot concentrator and thermodynamic model for the global redshift," Applied Physics Letters, 76, 9, 1197-1199, 2000.

4. R. Reisfeld, M. Eyal, V. Chernyak, R. Zusman, "Luminescent Solar Concentrators Based on Thin Films of Polymethylmethacrylate on a Polymethylmethacrylate Support", Solar Energy Materials, 17, 439-455, 1988.

5. A.R. Burgers, L.H. Sloof, R. Kinderman, J.A.M. Van Roosmalen, "Modelling of Luminescent Concentrators by Raytracing", Proceedings of $20^{\text {th }}$ European Photovoltaic Solar Energy Conference, Barcelona, Spain, 2005.

6. B. Rowan, S. McCormack, J. Doran, B. Norton, "Investigation of Various Geometries of Quantum Dot Solar Concentrator," Proceedings of 3rd Photovoltaic Science Application and Technology (PVSAT) Conference and Exhibition, Durham, U.K., 2007.

7. M. Sidrach de Cardona, M. Carrascosa, F. Meseguer, F. Cusso, F. Jaque, "Outdoor Evaluation of Luminescent Solar Concentrator Prototypes," Applied Optics, 24,13, 2028-2032, 1985.

8 G. Smestad, H. Ries, R. Winston, E. Yablonovitch, "The thermodynamic limits of light concentrators," Solar Energy Materials, 21, 99-111, 1990.

9. B. S Richards, A. Shalev, R.P. Corkish, “A Low Escape-Cone-Loss Luminescent Solar Concentrator”, $19^{\text {th }}$ European Photovoltaic Solar Energy Conference, Paris, 2004.

10. U. Rau, F. Einsele, G.C. Glaeser, "Efficiency limits of Photovoltaic Fluorescent Collectors," Applied Physics Letters, 87, 2005.

11 T. Markvart, "Detailed balance method for ideal single-stage fluorescent collectors", Journal of Applied Physics, 99 , 2006.

12 J. Goldschmidt, S.W. Glunz, A. Gombert, G. Willeke, "Advanced Fluorescent Concentrator", Proceedings $21^{\text {st }}$ European PV Conference, Dresden, Germany, 2006.

13. K. Heidler, A. Goetzberger, V. Witter, "Fluorescent Planar Concentrator. Monte-Carlo Computer Model, Limit Efficiency, and Latest Experimental Results", Proceedings $4^{\text {th }}$ EC PV Solar Energy Conference, Italy, 1982.

14. M. Carrascosa, S. Unamuno, F. Agullo-Lopez, "Simulation of the performance of PMMA luminescent solar collectors", Applied Optics, 22, 20, 3236-3241(1983).

15 S.J. Gallagher, P.C. Eames, B. Norton, "Quantum dot solar concentrator behaviour predicted using a ray trace approach", International Journal of Ambient Energy, 25, 1, 47-56, 2004.

16. M. Kennedy, B. Rowan, S. J. McCormack, J. Doran, B. Norton, "Modelling of a Quantum Dot Solar Concentrator and Comparison with Fabricated Devices", Proceedings $3^{\text {rd }}$ PV-SAT conference, Durham, U.K., 2007. 